\title{
AN ANALYSIS ON MORTALITY AMONG CALVES IN DANISH DAIRY HERDS
}

\author{
Flemming Skjøth and Lars Arne Hjort Nielsen \\ National Department of Cattle Husbandry; Udkærsvej 15; Skejby, DK-8200 Aarhus N, Denmark; \\ Phone.: 87405000; Fax: 87405010; Email address: fls@1r.dk
}

This study analysed the mortality among young calves born alive up to 180 days after birth, born in Danish dairy herds in 1998 and 1999. The objective of the study was to estimate the overall mortality and to analyse possible risk factors having an influence on the mortality.

The data material comprised 914,000 recorded births of dairy race cows in 9,333 dairy herds including the following animal level information: Dates of birth and possible death, sex, breed, change of herd, size at birth, calving eas (dystocier), calving number of mother and the following herd level information: Herd size, milk production level, indicator of organic farming status, indicator of major change in herd size, and county.

The effects of the animal and herd level factors on mortality in four periods after birth was investigated by a random effects model. The response being indicator of the calf dying during the period conditional on being alive at the beginning of the period. The four periods were defined as 1: 1 ? 14 days, 2: 15 ?28 days, 3: 29 ?56 days, and 4: 57 ?180 days after birth. The three main breeds (Danish Holstein, Red Danish, and Jersey) were analysed separately.

The observed overall mortality among heifers and bulls from day 1 to 180 after birth was $6.4 \%$, respectively $7.8 \%$. Among the animals not surviving 180 days a percentage of 45 died during the first 14 days. Although there is some variation between breeds, with Jersey having a higher level of mortality. The mortality of heifers is smaller than that of bulls. A small size of the calf at birth combined with a difficult delivery is increasing mortality, as well as the movement of an animal between herds increases its probability of not surviving.

The management on the farm is assumed to be confounded by the yield level, as farms with higher yield level has a reduced mortality. There is some variation in mortality between counties, which may be related to differences in production and housing systems, which are not registered yet.

As a conclusion, a number of production factors are documented to have an effect on calf mortality as well as some animal specific factors, which can help to focus on specific animals having an increased risk of dying prematurely. 